\title{
Detection of prostate cancer in unselected young men: prospective cohort nested within a randomised controlled trial
}

\author{
J Athene Lane, senior research fellow, Joanne Howson, lead research nurse, ${ }^{2}$ Jenny L Donovan, professor, ${ }^{1}$ \\ John R Goepel, consultant histopathologist, ${ }^{3}$ Daniel I Dedman, public health information specialist, ${ }^{4}$ \\ Liz Down, research associate, ${ }^{1}$ Emma L Turner, research associate, ${ }^{1}$ David E Neal, professor of surgical \\ oncology, ${ }^{5}$ Freddie C Hamdy, professor, head of urology and oncology ${ }^{2}$
}

'Department of Social Medicine, University of Bristo

${ }^{2}$ Academic Urology Unit,

Section of Oncology,

University of Sheffield,

Royal Hallamshire Hospital,

Sheffield, S10 2JF

${ }^{3}$ Sheffield Teaching Hospitals

NHS Foundation Trust,

Royal Hallamshire Hospital

${ }^{4}$ North West Public Health

Observatory, Liverpool John

Moores University, Liverpool

Oncology Centre, Addenbrooke's Hospital, Cambridge

Correspondence to: F C Hamdy

f.c.hamdy@sheffield.ac.uk

doi:10.1136/bmj.39381.436829.BE

\section{ABSTRACT}

Objective To investigate the feasibility of testing for prostate cancer and the prevalence and characteristics of the disease in unselected young men.

Design Prospective cohort nested within a randomised controlled trial, with two years of follow-up.

Setting Eight general practices in a UK city.

Participants 1299 unselected men aged 45-49.

Intervention Prostate biopsies for participants with a prostate specific antigen level of $1.5 \mathrm{ng} / \mathrm{ml}$ or more and the possibility of randomisation to three treatments for those with localised prostate cancer.

Main outcome measures Uptake of testing for prostate specific antigen; positive predictive value of prostate specific antigen; and prevalence of prostate cancer, TNM disease stage, and histological grade (Gleason score). Results 442 of 1299 men agreed to be tested for prostate specific antigen (34\%) and 54 (12\%) had a raised level.

The positive predictive value for prostate specific antigen was $21.3 \%$. Ten cases of prostate cancer were detected (2.3\%) with eight having at least two positive results in biopsy cores and three showing perineural invasion. One tumour was of high volume (cT2c), Gleason score 7, with a positive result on digital rectal examination; nine tumours were CT1c, Gleason score 6 , and eight had a negative result on digital rectal examination. Five of the nine eligible participants (55\%) agreed to be randomised. No biochemical disease progression in the form of a rising prostate specific antigen level occurred in two years of follow-up.

Conclusions Men younger than 50 will accept testing for prostate cancer but at a much lower rate than older men. Using an age based threshold of $1.5 \mathrm{ng} / \mathrm{ml}$, the

prevalence of prostate cancer was similar to that in older men $(3.0 \mathrm{ng} / \mathrm{ml}$ threshold) and some cancers of potential clinical significance were found.

Trial registration Current Controlled Trials ISRCTN20141297

\section{INTRODUCTION}

Prostate cancer is the commonest malignancy in males in many countries ${ }^{1}$ and screening for the disease is widespread - for example, in the United States around $60 \%$ of men over the age of 50 years are being tested annually. ${ }^{2}$ Screening for prostate cancer and the optimum treatment are controversial and three trials of screening are ongoing; the prostate, lung, colorectal and ovarian trial; the European randomised screening for prostate cancer trial; and the comparison arm of the prostate testing for cancer and treatment study (ProtecT). ${ }^{34}$ Three current trials of treatment are based on screen detected populations: the prostate testing for cancer and treatment study, ${ }^{5}$ the prostate cancer intervention versus observation trial, ${ }^{6}$ and the surveillance therapy against radical treatment trial. ${ }^{7}$ An earlier randomised trial with cases of clinically detected prostate cancer showed a survival benefit of surgery compared with watchful waiting, with a median follow-up of 8.2 years. $^{8}$ Extrapolation of these results to screen detected cases was, however, problematic because the cases were clinically detected and there was probably a lead time bias before the onset of symptoms of up to nine years. ${ }^{9}$ Therefore, in the absence of evidence from ongoing trials many countries (including the United Kingdom) have pragmatically agreed a policy whereby men aged 50 or more may have a test for prostate specific antigen after discussion with their general practitioner of the risks and benefits of testing. ${ }^{10}$

Men younger than 50 are usually offered the test only if they have a family history of the disease or are of black ethnicity. ${ }^{1011}$ Guidelines from the American National Comprehensive Cancer Network recommend screening for prostate specific antigen in men from age 40, with retesting either annually or at age 45 depending on the initial test value ${ }^{12}$ on the basis of findings in two retrospective cohorts that an increased antigen level in the fourth decade increased the risk of prostate cancer. ${ }^{1113}$ A recently published retrospective cohort study from Sweden reported a 3.7-fold increase in the odds ratio of detecting prostate cancer after 18 years for a $1 \mathrm{ng} / \mathrm{ml}$ increase in the prostate specific antigen result. ${ }^{14}$ These results were carefully validated because values for prostate specific antigen can decline 


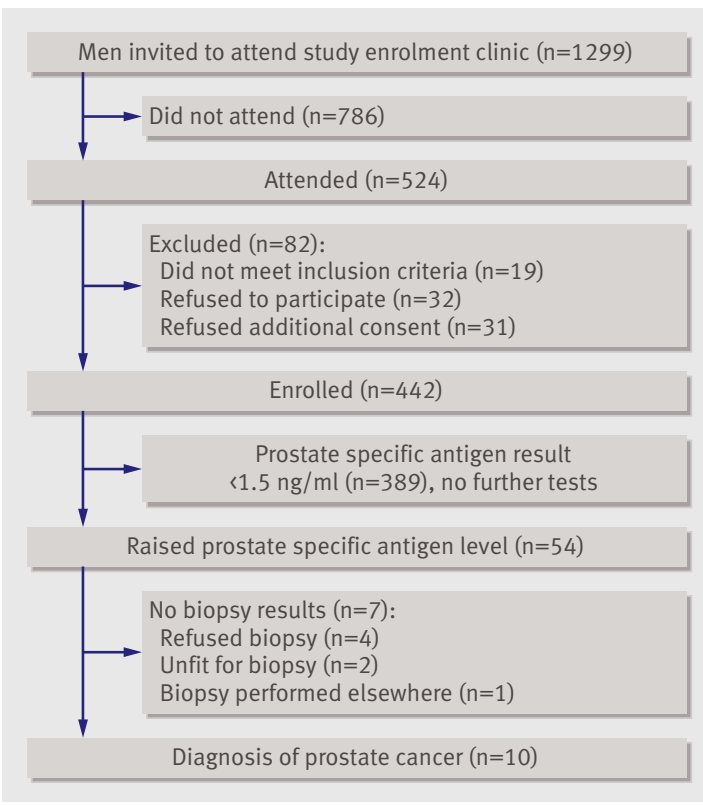

Fig 1 | Study profile

by up to $38 \%$ with incorrect processing and frozen storage. $^{14}$

Few population based studies of testing for prostate specific antigen have been done in men younger than 50. The prevalence of prostate cancer was $2 \%$ in a prospective cohort of 681 men aged 40-49 of African-American origin or with a family history of prostate cancer recruited in the United States. ${ }^{15}$ Prostate cancer was not found in two of the 44 Austrian blood donors with a prostate specific antigen value of at least $4.0 \mathrm{ng} / \mathrm{ml}$ who also had a suspicious result on digital rectal examination and a biopsy, but annual testing subsequently revealed six cases (detection rate 1.1\%). ${ }^{16}$ In a population based Austrian study, 28 of the 2054 men tested had a prostate specific antigen value of at least $2.5 \mathrm{ng} / \mathrm{ml}$ and three cases of prostate cancer were identified $(1.2 \%) .{ }^{17}$

In the prostate testing for cancer and treatment study, unselected men aged 50-69 have been invited for a prostate specific antigen test at nine UK centres since June 2001 to evaluate the effectiveness, cost effectiveness, and acceptability of treatments for clinically localised prostate cancer preceded by community based testing for prostate specific antigen. ${ }^{5}$ Using the same methods we carried out a nested study in men aged 45-49 to investigate the uptake of testing, the prevalence of prostate cancer, and characteristics of the disease.

\section{METHODS}

Since 2001 unselected men aged 50-69 and registered in randomly selected primary care centres in nine cities across the United Kingdom have been invited by letter to take part in the prostate testing for cancer and treatment (ProtecT) study. Full details are published elsewhere. ${ }^{518}$ Using the same methods, between November 2003 and August 2005 we carried out a nested study of men aged 45-49 and registered with eight general practices in Sheffield, in the north of England. The men were invited by letter to attend clinics for enrolment regardless of previous consultations or urological conditions (except those with major comorbidities that precluded enrolment in the trial). Study nurses explained the risks and benefits of testing for prostate specific antigen and provided details of the study. Eligible men who entered the study were tested. Participants had an additional consent form to return within 24 hours to authorise processing of the test. Participants gave written informed consent.

\section{Diagnostic procedures}

Participants with a prostate specific antigen value of 1.5 $\mathrm{ng} / \mathrm{ml}$ or more were invited for a transrectal ultrasound guided systematic prostate biopsy involving 10 core specimens, a repeat prostate specific antigen test, and a digital rectal examination. We selected a prostate specific antigen threshold for biopsy of $1.5 \mathrm{ng} / \mathrm{ml}$ to optimise detection of prostate cancer while reducing the number of unnecessary biopsies, on the basis of data from a Swedish cohort of younger men. ${ }^{14} \mathrm{~A}$ second biopsy was offered to those with either high grade prostatic intraepithelial neoplasia, atypical small acinar proliferation, a negative biopsy result but a persistently raised prostate specific antigen level, or a palpable abnormality on digital rectal examination. In addition, we offered annual tests to those men with a negative biopsy result. Men with an initial prostate specific antigen value of less than $1.5 \mathrm{ng} / \mathrm{ml}$ were not further tested. Participants with clinically localised prostate cancer were eligible for randomisation to radical three dimensional conformal radiotherapy, radical prostatectomy, or active monitoring (regular tests for prostate specific antigen and disease monitoring), with follow-up every 3-6 months for all arms according to treatment and study research protocols (mean follow-up 24 months).

\section{Outcome assessment and statistical analysis}

Research nurses recorded clinical and trial details on study databases. At enrolment, data were collected on participants' sociodemographic characteristics, including previous test results for prostate specific antigen that were checked against medical records. Ethnicity was based on categories from the 1991 UK census. Specialist uropathologists reported the biopsy and disease results. We classified the results of digital rectal examinations as positive if there was a palpable abnormality. Tumours were assessed by histological grading using the Gleason scoring system (6-10); tumour staging using the 2002 TNM classification; and a nomogram for predicting indolent disease in men aged 50 or more. ${ }^{19}$ We calculated the positive predictive value of the prostate specific antigen test, 
with the biopsy as the reference test. All analyses were done using Stata version 9.

\section{RESULTS}

Overall, 524 of 1299 unselected men (40\%) aged 45-49 invited attended a study enrolment clinic; 473 (36.4\%) were included in the study (fig 1). Thirty two chose not to be tested $(2 \%)$ and $19(1 \%)$ were excluded for other reasons: 16 were medically unsuitable for the cancer treatments, two could not give informed consent, and one was outside the age range. Four hundred and forty two participants $(34 \%)$ gave additional consent to process their prostate specific antigen test.

The mean age of participants was 48 years and 413 were white $(98 \%)$. Ten of the participants had prostate cancer. These participants were white and did not report a family history of the disease compared with 22 of $422(5 \%)$ participants without cancer. None of the 18 participants who had been previously tested for prostate specific antigen (4\%) had a diagnosis of prostate cancer. Three hundred and fifty three participants $(97 \%)$ reported that urinary symptoms had little or no effect on their life.

\section{Detection of prostate cancer}

The mean value for prostate specific antigen was 0.9 (SD 0.75 ) $\mathrm{ng} / \mathrm{ml}$ and the median was $0.7 \mathrm{ng} / \mathrm{ml}$ (fig 2). Fifty four participants had raised levels of prostate specific antigen $(12.2 \%$ of those enrolled) and 47 underwent a biopsy (87\%): four declined, two were unfit for the procedure, and results for one who had a biopsy elsewhere were unavailable. Twelve participants had repeat biopsies after consultation with the urologist and two after findings of high grade prostatic intraepithelial neoplasia. In those men who did not have a further biopsy after an initial negative biopsy result, 10 were referred back to their general practitioners, five declined a biopsy, one had an unavailable result, and eight elected to undergo further testing at 12 months.

Nine cases of prostate cancer were detected at the first biopsy and one at the second and all the initial values for prostate specific antigens were less than 4.0 $\mathrm{ng} / \mathrm{ml}$. The detection rate for prostate cancer was $2.3 \%$ and the prevalence of disease in participants with a raised level of prostate specific antigen was 21.3\% (95\% confidence interval $7.4 \%$ to $29.6 \%$ ). The positive predictive value of the prostate specific antigen test was $21.3 \%$ (25.6\% when corrected for participants who did not undergo a biopsy).

\section{Clinical and disease features of the cancers}

Eight participants had two or more positive biopsy core results and five had four or more positive results. The total tumour length ranged from less than $0.5 \mathrm{~mm}$ to $24 \mathrm{~mm}$, with a maximum of $13 \mathrm{~mm}$ in a single core (table). Perineural invasion was reported in three cases. Nine participants had cT1c tumours, Gleason score 6, of whom eight had a negative digital rectal examination result (one result was unavailable). One participant had a cT2c tumour, Gleason score 7 $(3+4)$, and a positive digital rectal examination result. Five cases were classified as potentially indolent using a nomogram and five were classified as of potential clinical significance. ${ }^{19}$

Five participants agreed to be randomised (55\%); one to active monitoring and two each to radiotherapy and surgery. One participant randomised to surgery rejected the allocation and selected radiotherapy. Two participants chose active monitoring, one radiotherapy, one brachytherapy. The comorbidities of one participant precluded randomisation and he received radiotherapy. Disease stage was organ confined with no evidence of nodal metastases (pT2aN0Mx) for the participant who received a radical prostatectomy, with a Gleason score of $6(3+3)$. The surgical margins were negative with no evidence of

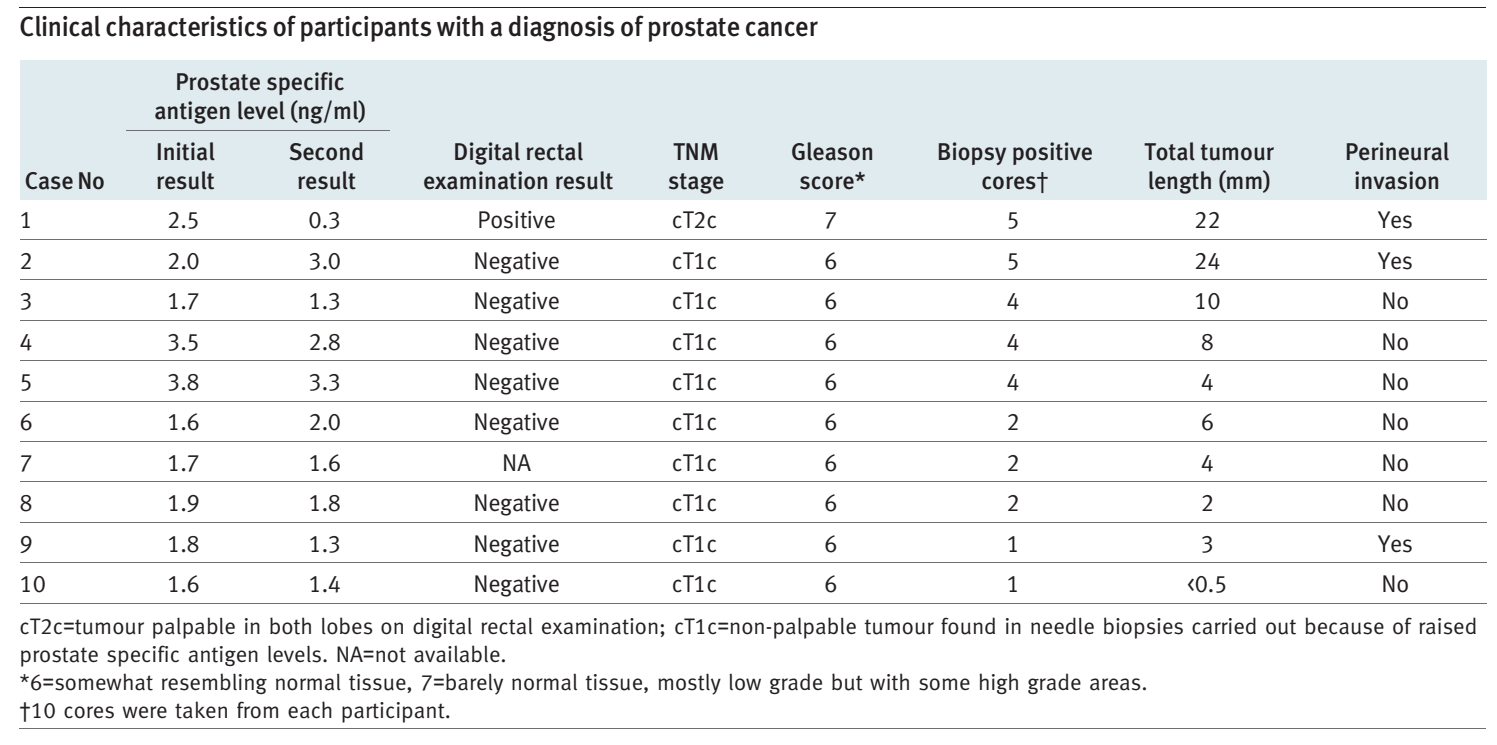


extracapsular or perineural spread of the disease. No biochemical failure in the form of a rising prostate specific antigen was detected in nine of the 10 cases over a mean of 24 months' follow-up (one participant was lost to follow-up).

\section{DISCUSSION}

The uptake of prostate specific antigen testing within the context of a clinical trial in unselected men aged $45-49$ was $34 \%$ and the prevalence of prostate cancer was $2.3 \%$, with five of the 10 cancers classified as potentially clinically significant.

Men aged 45-49 had a significantly lower uptake of testing $(34.0 \%$, 95\% confidence interval $31.2 \%$ to $36.3 \%)$ compared with those aged 50-69 (50\%, 49.7\% to $50.3 \%$ ) in the main prostate testing for cancer and treatment study. ${ }^{20}$ The uptake of testing was 25\%-46\% in the European randomised screening for prostate cancer trial with older participants and $32 \%$ in an Austrian study with younger men. ${ }^{317}$ The American high risk cohort of younger men was recruited by using a press release so the response rate is unknown. ${ }^{15}$

The detection rate for prostate cancer in the prostate testing for cancer and treatment study was broadly similar in younger $(2.3 \%, 0.9 \%$ to $3.7 \%)$ and older men $(2.9 \%, 2.7 \%$ to $3.0 \%) .{ }^{20}$ If all participants had been offered biopsies using the same prostate specific antigen threshold, however, a greater difference may have emerged, as the threshold for the older men was $3.0 \mathrm{ng} / \mathrm{ml}$ but prostate specific antigen values between 2.0 and $3.0 \mathrm{ng} / \mathrm{ml}$ are also associated with a slightly increased risk of prostate cancer. ${ }^{21}$ The prevalence of prostate cancer reported here was higher than the Austrian studies in younger men ${ }^{16}{ }^{17}$ and comparable to the American high risk cohort. ${ }^{15}$ However, we used systematic 10 core biopsies (adding two lateral biopsies on each side) in this study, which are more optimal for cancer detection than the previously used sextant biopsy scheme (three biopsies from each side including base, middle, and apex of the prostate). ${ }^{22}$ Data on prevalence were also less accurate in previous cohort studies as only about $50 \%$ of men with a raised prostate specific antigen level had a biopsy. ${ }^{1517}$ It is also noteworthy that those men with the highest values for prostate specific antigen were not found to have cancer (fig 2 and table), confirming the low specificity of the test.

The prognosis of prostate cancers detected by screening is a critical problem with results from the European randomised screening for prostate cancer trial indicating that about half of the cancers detected are clinically indolent. ${ }^{19}$ Half the cancers were categorised as potentially indolent in our study, although the nomogram used in the European trial has yet to be validated in this age range. All cases were clinically localised in the younger men compared with $76 \%$ in men aged 50-69 in the prostate testing for cancer and treatment study, ${ }^{20}$ but more accurate assessment of disease data was not possible in those

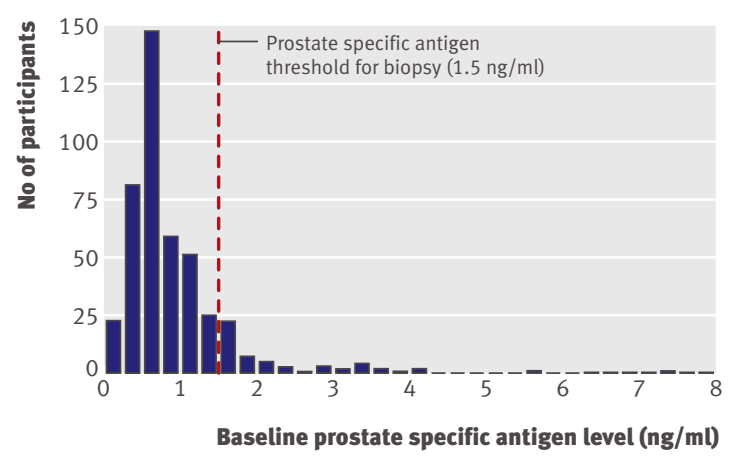

Fig 2 | Distribution of prostate specific antigen

receiving non-surgical treatments. The 26 cancers detected in the American study were clinically localised, with a mean Gleason score of 6 , whereas disease staging showed that 18 of 24 cases $(78 \%)$ were localised tumours, with a mean Gleason score of $6 .{ }^{23}$ Biochemical disease progression in the form of a rising prostate specific antigen occurred in six cases (24\%) over two years' follow-up, but this short period did not permit comparison of outcome between younger and older men. ${ }^{24}$ In the smaller Austrian study, five detected tumours were localised and Gleason scores ranged from $4-8 .{ }^{16}$ None of the prospective cohorts of younger men yet has sufficient follow-up to inform the debate on the long term outcome of these cancers detected by testing for prostate specific antigen. ${ }^{24}$ In the only outcome data published in men younger than 50, survival without biochemical relapse (in the form of rising prostate specific antigen) after radical prostatectomy seemed better in younger men. ${ }^{25}$

Our study has several strengths but also some limitations. The major strength is the prospective design using an unselected population with minimal contamination of the prevalence data by previous testing for prostate specific antigen, which is not routinely recommended in the United Kingdom. ${ }^{10}$ Study results were also enhanced by the high uptake for biopsy, the use of a systematic 10 core biopsy protocol, detailed histology, and standardised diagnosis algorithms.

The limitations were that testing was done within the context of a clinical trial, which, along with trial exclusion criteria, probably reduced uptake. Enrolment in clinical trials is higher in older age groups, ${ }^{26}$ probably because of the perceived additional demands of time and visits to the research centre. Although the study was population based there may have been self selection criteria by participants who may have attended because of minor health problems, general anxiety, or misconceptions about the disease and its implications, but these were not related to urinary symptoms, which were infrequently reported. It is not possible to examine the characteristics of the non-responders because of the UK Data Protection Act 1998 . The study population was predominately white (reflecting the population of the catchment's 


\section{WHAT IS ALREADY KNOWN ON THIS TOPIC}

Screening for prostate cancer, when undertaken, usually starts at age 50 , in the absence of risk features

A lower age limit has recently been adopted in the United States on the basis of two retrospective cohorts, which found that prostate specific antigen levels in the fourth decade predicted prostate cancer

\section{WHAT THIS STUDY ADDS}

The uptake of testing for prostate specific antigen by men aged $45-49$ was $34 \%$ and the prevalence of prostate cancer was $2.3 \%$

Younger men were less likely to subscribe to testing than those older than 49

One in two cancers detected is potentially clinically important but the prognosis is currently unknown negative biopsy results, and the risks of overtreatment and associated side effects to those with a diagnosis of cancer.

Some of these issues may be resolved by the development of robust prognostic nomograms and biomarkers to reliably identify clinically significant disease. Furthermore, randomised trials currently under way should resolve the controversies around the testing for and treatment of prostate cancer. This study will inform the debate about thresholds for prostate specific antigen and age limits only if screening for prostate cancer is proved to be effective in these ongoing trials.

We thank the research group of the prostate testing for cancer and treatment study for their contribution and Richard Martin for his comments.

area) so the results may not be applicable to other ethnic groups where the prevalence of prostate cancer differs. $^{10}$ Finally, we could not determine the performance of the prostate specific antigen test in this age range (beyond the positive predictive value) or the population prevalence of prostate cancer as biopsies were not offered to all participants.

Previous long term studies in men mostly younger than 50 included two retrospective cohorts with stored serum and at least 10 years' follow-up, which showed that baseline levels of prostate specific antigen were predictive of risk for prostate cancer. ${ }^{113}$ Degradation of prostate specific antigen in archived serum, however, can introduce measurement bias in predicting prostate cancer unless specific procedures are used for serum processing and storage. ${ }^{14}$ Markov modelling suggested a benefit on mortality from prostate cancer in screening men at age 40 and 45 with biennial testing from age 50-75, although no account was taken of the uptake of screening. ${ }^{27}$

Potential benefits and harms of prostate cancer testing This study provides evidence to inform the debate about testing and screening for prostate cancer in men aged less than 50. Firstly, it has shown that men invited to testing will attend, but at a much lower rate than older men, and so if screening was introduced greater efforts would have to be made to maximise uptake in this age group. Secondly, it has confirmed that a prostate specific antigen threshold of $1.5 \mathrm{ng} / \mathrm{ml}$ results in a comparable detection rate to that in older men with a threshold of $3.0 \mathrm{ng} / \mathrm{ml}$. At present, however, it is not possible to determine which tumours would result in clinically significant disease and which represent indolent disease.

One study ${ }^{12}$ advocated that screening for prostate cancer should start at age 45. If the 2236000 men aged 45-49 in the UK population (UK Office of National Statistics, 2004) were to undergo screening for prostate specific antigen it can be projected from our data that 272905 men would have a raised prostate specific antigen level and, of these, 51449 would have prostate cancer. Some of these cancers may benefit from treatment although this has to be set against the possible distress caused to the 221456 men with
Contributors: JAL, JLD, DEN, and FCH had full access to the data in the study. JAL is the trial coordinator. JLD, DEN, and FCH are the principal investigators. $J H$, JRG, and FCH carried out the substudy. DJD, LD, and ELT contributed to data collection and analysis. JAL wrote the first draft and together with FCH wrote the final manuscript. All authors contributed to the intellectual content of the draft manuscript and approved the final version. JAL is guarantor of the paper. Funding: The prostate testing for cancer and treatment study is funded and sponsored by the Health Technology Assessment programme (projects 96/20/06 and 96/20/99). The views and opinions expressed are those of the authors and do not necessarily reflect those of the Department of Health.

Competing interests: None declared.

Ethical approval: This study was approved by Trent multicentre research ethics committee.

Provenance and peer review: Not commissioned; externally peer reviewed.

1 Giovannucci E, Platz EA. Epidemiology of prostate cancer. Comprehensive textbook of genitourinary oncology. Lippincott: Williams and Wilkins, 2006:9-21.

2 Weir HK, Thun MJ, Hankey BF, Ries LA, Howe HL, Wingo PA, et al. Annual report to the nation on the status of cancer, 1975-2000, featuring the uses of surveillance data for cancer prevention and control. J Natl Cancer Inst 2003;95:1276-99.

3 De Koning HJ, Auvinen A, Berenguer SA, Calais DS, Ciatto S, Denis L, et al. Large-scale randomized prostate cancer screening trials: program performances in the European randomized screening for prostate cancer trial and the prostate, lung, colorectal and ovary cancer trial. Int J Cancer 2002;97:237-44.

4 Donovan JL, Martin R. Commentary: screening for prostate cancer. Int J Epidemiol 2007;36:30-1.

5 Donovan JL, Hamdy F, Neal DE, Peters TJ, Oliver SE, Brindle L, et al. Prostate test for cancer and treatment (ProtecT) feasibility study. Health Technol Assess 2003; 7:88.

6 Wilt TJ, Brawer MK. The prostate cancer intervention versus observation trial: a randomized trial comparing radical prostatectomy versus expectant management for the treatment of clinically localized prostate cancer. J Urol 1994;152:1910-4.

7 Klotz L. Active surveillance for prostate cancer: for whom? J Clin Oncol 2005;23:8165-9.

8 Bill-Axelson A, Holmberg L, Ruutu M, Haggman M, Andersson SO, Bratell S, et al. Radical prostatectomy versus watchful waiting in early prostate cancer. N Engl J Med 2005;352:1977-84.

9 Draisma G, Boer R, Otto SJ, van der Cruijsen IJ, Damhuis RAM, Schroder FH, et al. Lead times and overdetection due to prostatespecific antigen screening: estimates from the european randomized study of screening for prostate cancer. J Natl Cancer Inst 2003;95:868-78. information on the outcome of screening and estimates of ad hoc screening with particular reference to England and Wales. BJU Int 2005;95(suppl 3):4-15.

11 Whittemore AS, Cirillo PM, Feldman D, Cohn BA. Prostate specific antigen levels in young adulthood predict prostate cancer risk: results from a cohort of black and white Americans. J Urol 2005;174:872-6.

12 Catalona WJ, Loeb S, Han M. Viewpoint: expanding prostate cancer screening. Ann Intern Med 2006;144:441-3. levels of prostate-specific antigen predict long-term risk of prostate
10 Melia J. Part 1: The burden of prostate cancer, its natural history,

13 Fang J, Metter EJ, Landis P, Chan DW, Morrell CH, Carter HB. Low 
cancer: results from the Baltimore longitudinal study of aging. Adult Urol 2001;58:411-6.

14 Lilja H, Ulmert D, Bjork T, Becker C, Serio AM, Nilsson J-A, et al. Long-term prediction of prostate cancer in a large, representative Swedish cohort: prostate kallikreins measured at age 44-50 predict prostate cancer up to 25 years before diagnosis. J Clin Oncol 2007;25:431-6.

15 Catalona WJ, Antenor JAV, Roehl KA. Screening for prostate cancer in high risk populations. J Urol 2002;168:1980-4.

16 Reissigl A, Pointner J, Horninger W, Strasser H, Mayersback P, Klocker $\mathrm{H}$, et al. PSA-based screening for prostate cancer in asymptomatic younger males: pilot study in blood donors. Prostate 1997:30:20-5.

17 Reissigl A, Pointner J, Horninger W, Ennemoser O, Strasser H, Klocker $\mathrm{H}$, et al. Comparison of different prostate-specific antigen cutpoints for early detection of prostate cancer: results of a large screening study. Urology 1995;46:662-5.

18 Donovan J, Mills N, Smith M, Brindle L, Jacoby A, Peters T, et al. Quality improvement report: improving design and conduct of randomised trials by embedding them in qualitative research: ProtecT (prostate testing for cancer and treatment) study. Commentary: presenting unbiased information to patients can be difficult. BMJ 2002;325:766-70.

19 Roemeling S, Steyerberg EW, Roobol MJ, Wolters T, Gosselaar C, Bangma $\mathrm{CH}$, et al. Nomogram use for the prediction of indolent prostate cancer in men selected for active surveillance. Eur Urol Suppl 2007;6:1026.

20 Lane A, Hamdy F, Neal D, Donovan J. The protect trial-evaluating the effectiveness of treatment for clinically localised prostate cance (ISRCTN20141297). Br J Cancer 2004;91(suppl 1):S80.
21 Thompson IM, Pauler DK, Goodman PJ, Tangen CM, Lucia MS, Parnes HL, et al. Prevalence of prostate cancer among men with a prostate-specific antigen level < or $=4.0 \mathrm{ng}$ per milliliter. $N$ Engl J Med 2004;350:2239-46.

22 Eichler K, Hempel S, Wilby J, Myers L, Bachmann LM, Kleijnen J. Diagnostic value of systematic biopsy methods in the investigation of prostate cancer: a systematic review. J Urol 2006;175:1605-12.

23 Loeb S, Gonzalez CM, Roehl KA, Han M, Antenor IA, Yap RL, et al. Pathological characteristics of prostate cancer detected through prostate specific antigen based screening. J Urol 2006; 175:902-6.

24 Loeb S, Roehl KA, Catalona WJ, Nadler RB. Prostate specific antigen velocity threshold for predicting prostate cancer in young men. IUrol 2007;177:899-902.

25 Smith CV, Bauer JJ, Connelly RR, Seay TM, Kane C, Foley J, et al. Prostate cancer in men age 50 years or younger: a review of the Department of Defense Center for Prostate Disease Research multicenter prostate cancer database. J Urol 2000;164:1964-7.

26 Lane A, Harvey R, Murray L, Harvey I, Donovan J, Nair P, et al. A placebo-controlled randomized trial of eradication of Helicobacter pylori in the general population: study design and response rates of the Bristol helicobacter project. Control Clin Trials 2002;23:321-32.

27 Ross KS, Ballentine Carter H, Pearson JD, Guess HA. Comparative efficiency of prostate-specific antigen screening strategies for prostate cancer detection. JAMA 2000;284:1399-405.

Accepted: 26 September 2007 